\title{
Silencing HO-1 sensitizes SKM-1 cells to apoptosis induced by low concentration 5-azacytidine through enhancing p16 demethylation
}

\author{
PING WANG ${ }^{1-3}$, DAN MA $^{1,3,4}$, JISHI WANG ${ }^{1-3}$, QIN FANG $^{4}$, RUI GAO ${ }^{1-3}$, \\ WEIBING WU ${ }^{1-3}$, TANGSHENG $\mathrm{LU}^{5}$ and $\mathrm{LU} \mathrm{CAO}^{5}$ \\ ${ }^{1}$ Department of Hematology, ${ }^{2}$ Guizhou Province Hematopoietic Stem Cell Transplantation Center, \\ Affiliated Hospital of Guiyang Medical University; ${ }^{3}$ Key Laboratory of Hematological Disease Diagnostic \\ and Treat Centre of Guizhou Province, Guiyang 550004; ${ }^{4}$ Department of Pharmacy, \\ Affiliated Baiyun Hospital of Guiyang Medical University, Guiyang 550014; \\ ${ }^{5}$ School of Pharmacy, Guiyang Medical University, Guiyang 550004, P.R. China
}

Received November 4, 2014; Accepted December 29, 2014

DOI: $10.3892 /$ ijo.2015.2835

\begin{abstract}
Heme oxygenase-1 was reported previously as a resistance target on acute myelocytic leukemia (AML). We found that $\mathrm{HO}-1$ was resistant to 5-azacytidine (AZA) treatment of myelodysplastic syndrome (MDS), and explored further the relative mechanisms. Patient bone marrow mononuclear cells $(n=48)$ diagnosed as different levels of MDS were collected. Cell growth was evaluated by MTT assay; cell cycle and apoptosis were detected by flow cytometry; mRNA expression was assessed by real-time PCR, protein expression was analyzed through western blotting. Methylation was assessed by MSP. The survival time, and weight of mice were recorded. HO-1 overexpression was observed in SKM-1 cells after AZA treatment comparing to other cell lines. The HO-1 expression in MDS patients with high-risk was higher than in low-risk patients. After HO-1 was silenced by lentivirus-mediated siRNA, the proliferation of SKM-1 cells was effectively inhibited by low concentration AZA, and the cell cycle was arrested in the $\mathrm{G} 0 / \mathrm{G} 1$ phase. Upregulation of p16 and changing of p16-relative cell cycle protein was observed after silencing HO-1 in AZA treated SKM-1 cells. In addition, DNMT1 was downregulated following the decrease of HO-1 expression. In vivo, silencing HO-1 inhibited SKM-1 cell growth induced by AZA in a NOD/SCID mouse model. Silencing HO-1 sensitized SKM-1 cells toward AZA, which may be attributed to the influence of HO-1 on AZA-induced p16 demethylation. HO-1 may be one of the targets that enhance the therapeutic effects of AZA on MDS malignant transformation inspiring
\end{abstract}

Correspondence to: Professor Jishi Wang, Department of Hematology, Affiliated Hospital of Guiyang Medical University, Guiyang 550004, P.R. China

E-mail: wangjishi9646@163.com

Key words: myelodysplastic syndrome, SKM-1, 5-azacytidine, heme oxygenase-1, p16 new treatment methods for high-risk and very high-risk MDS patients in clinical practice.

\section{Introduction}

Myelodysplastic syndrome (MDS), as a heterogeneous group of related clonal diseases, is typified by monolineage or multilineage dysplasia, ineffective hematopoiesis, or high risk of transformation to acute myelocytic leukemia (AML) (1). MDS has been associated with aberrant methylation of relevant gene promoters that can facilitate tumor onset by silencing anti-oncogenes and by changing the expressions of tumor-related genes $(2,3)$. Unlike genetic mutation, these epigenetic changes can be reverted by drugs such as DNA methyltransferase inhibitor 5-azacytidine (AZA) that targets the treatment of AML/MDS. Compared with traditional chemotherapeutic agents, AZA is able to significantly increase the overall survival rates of medium-risk II, high-risk MDS and WHO-AML patients (4).

As a cytosine nucleoside analogue, AZA results in demethylation at low concentration while endows cytotoxicity at high concentration (5). Low-concentration AZA weakens the methylation of $\mathrm{CpG}$ islands in anti-oncogene promoter regions by inhibiting methyltransferase, thus promoting the expressions of anti-oncogenes (e.g., p15, p16 and other negative cell cycle regulatory genes). However, it barely toxifies normal cells (6). Although AZA evidently raises the overall survival rates of medium-risk II, high-risk MDS and WHO-AML patients, the rate of complete remission remains low $(7,8)$. Heme oxygenase-1 (HO-1), which is an isozyme of heme oxygenase, has been observed in many solid tumors including melanoma, brain tumor and lymphosarcoma (9). Previous studies also revealed that HO-1 affects the response of leukemic cells to chemotherapy as well as their proliferation and apoptosis via the TNF and NF- $\kappa \mathrm{B}$ pathways (10). High HO-1 expression may indicate progression, poor prognosis and chemotherapy resistance of leukemia (11), which protects normal bone marrow cells from the side effects of chemotherapy but allows 
tumor cells to resist drugs (12). Recent studies have reported that demethylation of Nrf2 induced by certain drugs led to an increase in the protein expression and activity of heme oxygenase-1, including 5-fluorouracil and dietary phytochemicals $(13,14)$. Nevertheless, neither the role of HO-1 in MDS nor in the demethylating effect of AZA has been well studied hitherto. Our study found that after being treated with low concentration AZA $(0.5 \mu \mathrm{M})$, SKM-1 cells expressed more $\mathrm{HO}-1$, and the bone marrow mononuclear cells (MNCs) from high-risk and very high-risk MDS patients also expressed more HO-1 than those from low-risk and very low-risk MDS patients did. Considering the role of HO-1, we hypothesized that high HO-1 expression may weaken the therapeutic effects of AZA and promote MDS malignant progression.

Therefore, we analyzed the influence of $\mathrm{HO}-1$ on AZA-induced proliferation inhibition, apoptosis, cell cycle arrest and demethylation in SKM-1 cells by silencing HO-1 gene with lentivirus-mediated siRNA and by upregulating it with Hemin $(15 \mu \mathrm{mol} / \mathrm{l})$. Furthermore, the possible mechanism was explored.

\section{Materials and methods}

Cell lines and cell culture conditions. Human AML cell lines, including HEL, U937 and THP-1, were propagated in a monolayer culture in RPMI-1640 medium. MDS cell line SKM-1 was purchased from the Japanese Collection of Research Bioresources. RPMI-1640 medium was supplemented with $15 \%$ fetal bovine serum, $100 \mathrm{U} / \mathrm{ml}$ penicillin, and $100 \mathrm{mg} / \mathrm{ml}$ streptomycin. The medium and antibiotics were bought from Invitrogen (Carlsbad, CA, USA). All cells were maintained in a $37^{\circ} \mathrm{C}$ incubator with $95 \%$ humidity and $5 \% \mathrm{CO}_{2}$.

Patient samples. Bone marrow samples were collected during routine diagnostic assessment after written informed consent had been obtained. The patients were diagnosed by using WHO classification. Patients' bone marrow MNCs were separated by Ficoll-Hypaque (Sigma Chemical Co.) densitygradient centrifugation and used immediately. All participants provided written informed consent prior to entering the study. The study was approved by the Institutional Review Board of the Affiliated Hospital of Guiyang Medical College.

Chemicals and antibodies. AZA (99.0\% purity) was obtained from the Shanghai Huilun Life Science and Technology Corp. Antibodies for western blot analysis were obtained from Cell Signaling Technology (Beverly, MA, USA) and secondary antibodies were purchased from Li-Cor Corp. (Lincoln, NE, USA).

Cell proliferation assay. Cells were seeded at a density of 1,000 cells/well in a 96 -well plate. After overnight incubation at $37^{\circ} \mathrm{C}$, serial dilutions of test compounds were added and the cells were further incubated in $5 \% \mathrm{CO}_{2}$ for $24 \mathrm{~h}$ at $37^{\circ} \mathrm{C}$. The inhibitory effects were determined using colorimetric 3-(4,5-dimethylthiazol-2-yl)-2,5-diphenyltetrazolium bromide (MTT) assay (MTT; Sigma, USA).

Apoptosis analysis. Apoptotic cells were analyzed by flow cytometry with propidium iodide (PI) staining (BD
Biosciences, San Jose, CA, USA). Cells were treated with fresh drug preparations and medium for $24 \mathrm{~h}$, washed in PBS and resuspended in $100 \mu \mathrm{l}$ of binding buffer containing $5 \mu \mathrm{l}$ of Annexin V (BD Pharmingen, San Diego, CA, USA). The cells were analyzed by flow cytometry after adding $5 \mu \mathrm{l}$ of PI. Annexin V binds to cells that express phosphatidylserine on the outer layer of the cell membrane, while PI stains the cellular DNA of those cells with a compromised cell membrane. This allows viable cells (unstained with either fluorochrome) to be distinguished from apoptotic cells (stained only with Annexin V) and necrotic cells (stained with both Annexin V and PI). After being stained at room temperature for $15 \mathrm{~min}$ in dark, the cells were measured by flow cytometry and Cell Quest software (BD Biosciences). All experiments were conducted at least three times.

Cell cycle analysis. Cell cycle was determined by flow cytometry using PI. Cells $\left(10^{5} / \mathrm{ml}\right)$ were washed with PBS and fixed with $70 \%$ ice-cold ethanol. After $2 \mathrm{~h}$, the cells were washed twice in PBS and resuspended in PBS containing $50 \mathrm{mg} / \mathrm{ml}$ PI (Sigma), $200 \mathrm{mg} / \mathrm{ml}$ DNase free RNase A (Citomed), and $0.1 \%$ Triton $\mathrm{X}-100$ for $1 \mathrm{~h}$ at room temperature. Acquisition was performed on a FACS Calibur flow cytometer (Becton-Dickinson). Data were analyzed with the cell cycle program from FlowJo software (Tree Star, Inc. Ashland, OR, USA).

Quantitative real-time PCR. Total RNA was isolated and purified from cells using the RNeasy kit (Qiagen, Hilden, Germany) and reverse-transcribed using the Omniscript Reverse Transcription kit (Qiagen). cDNAs were analyzed by quantitative real-time PCR using primers provided by Airui Technology Corp. (Guiyang, China) and iQ SYBR Green supermix (Bio-Rad, Hercules, CA, USA).

Methylation-specific PCR. Genomic DNA was prepared from cells and then subjected to bisulfite conversion. The methylation status of $\mathrm{CpG}$ islands in the p16 gene promoter was determined by methylation-specific PCR (MSP). The primers used for unmethylated p16 were: sense, 5'-TTTTTGGTGTTA AAGGGTGGTGTACT-3' and antisense, 5'-CACAAAAACC CTCACTCACAACAA-3', which yielded a fragment of $132 \mathrm{bp}$. The primers used for methylated p16 were: sense, 5'-GTGTTA AAGGGCGGCGTAGC-3' and antisense, 5'-AAAACCCTCA CTCGCGACGA-3', which yielded a PCR product of $122 \mathrm{bp}$. DNA was amplified according to the following protocol: $95^{\circ} \mathrm{C}$ for $5 \mathrm{~min}$, followed by 40 cycles at $95^{\circ} \mathrm{C}$ for $1 \mathrm{~min}, 60^{\circ} \mathrm{C}$ for $30 \mathrm{sec}, 72^{\circ} \mathrm{C}$ for $1 \mathrm{~min}$, and a final extension step at $72^{\circ} \mathrm{C}$ for $10 \mathrm{~min}$. Amplified products were resolved on $3 \%$ agarose gels and visualized under ultraviolet light after staining with ethidium bromide. Results were confirmed by repeating MSP assays after an independently performed bisulfite treatment.

Western blot analysis. Western blot analysis was carried out to analyze protein expression and activation after cells were treated with AZA alone or AZA plus Hemin, or after specific HO-1 knockdown. Briefly, cells were washed in PBS, collected, and then lysed in radioimmunoprecipitation assay buffer $(50 \mathrm{mmol} / 1$ Tris- $\mathrm{HCl} ; 150 \mathrm{mmol} / 1 \mathrm{NaCl} ; 0.1 \%$ SDS; $0.5 \%$ Na-deoxycholate; $1 \%$ NP40) containing proteinase 
Table I. Patient characteristics.

\begin{tabular}{lc}
\hline Characteristics & $(\mathrm{n}, \%)$ \\
\hline Age(years), $\mathrm{n}$ & 48 \\
$<70$ & $34(71)$ \\
$\geq 70$ & $14(29)$ \\
Sex, $\mathrm{n}$ & 48 \\
Male & $37(77)$ \\
Female & $11(23)$ \\
WHO Classification, $\mathrm{n}$ & 48 \\
RA/RAS & $10(21)$ \\
RCMD/RSCMD & $14(29)$ \\
RAEB-I & $4(8)$ \\
RAEB-II & $17(36)$ \\
RAEB-t/AML & $3(6)$ \\
WPSS risk group & 48 \\
Very low & $9(19)$ \\
Low & $12(25)$ \\
Intermediate & $7(14)$ \\
High & $11(23)$ \\
Very high & $9(19)$ \\
\hline
\end{tabular}

RA, refractory anemia; RCMD, refractory cytopenia with multilineage dysplasia; RAEB, RA with excess of blasts; RAEB-t, RA with excess of blasts in transformation; AML, acute myeloid leukemia; WPSS, WHO Prognostic Score System; WHO, World Health Organization.

inhibitor cocktail and phosphatase inhibitor cocktail (Roche Applied Science, Indianapolis, IN, USA). The lysate was centrifuged at $10000 \mathrm{x} \mathrm{g}$ at $4^{\circ} \mathrm{C}$ for $10 \mathrm{~min}$. The supernatant (50-100 $\mathrm{mg}$ protein) was fractioned by SDS-PAGE using $10 \%$ gels and was transferred electrophoretically to Hybondenhanced chemiluminescence membranes (GE Healthcare Life Sciences, Piscataway, NJ, USA). The membrane was blocked with blocking buffer (Li-Cor Corp.) at room temperature for $1 \mathrm{~h}$ and then incubated with the primary antibody at $4^{\circ} \mathrm{C}$ overnight. After being washed by PBS with $0.1 \%$ Tween-20 (PBST), the membrane was incubated with the IRDye infrared secondary antibody (Li-Cor Corp.) for $1 \mathrm{~h}$ at room temperature, washed with PBST again and detected with enhanced chemiluminescence.

The recombinant lentiviral vector and transfection. Selfprepared recombinant lentivirus-V5-D-TOPO-HO-1-vector and control vector lentivirus-V5-D-TOPO-vector were cotransfected into the 293FT packaging cell line. The supernatant was collected 48 and $72 \mathrm{~h}$ after infection to harvest the recombinant virus. Lentivirus-V5-DTOPO-vector and lentivirus-V5-D-TOPO- vector -HO-1 were cotransfected into SKM-1 cells. The transfection rate was determined by western blot analysis.

Intravenous MDS model of NOD/SCID mice. NOD/SCID mice, purchased from Beijing laboratory animal center, were injected intraperitoneally with $150 \mathrm{mg} / \mathrm{kg}$ cyclophosphamide (Wako Pure Chemical Industries, Kyoto, Japan) on each of two consecutive days to repress residual immunity. The day after the third cyclophosphamide injection, mice were randomized into three groups, SKM-1 (without treatment), SKM-1-vector (transfected with empty vector) and SKM-1siHO-1 (transfected with siRNA targeting HO-1) cells $\left(3 \times 10^{7}\right.$ cells per animal) were separately injected intravenously into the mice tail vein of the three groups. On the sixth day after inoculation (day 0), the three groups were further grouped into six: SKM-1, SKM-1-vector, SKM-1-siHO-1, SKM-1 (AZA), SKM-1-vector (AZA), SKM-1-siHO-1 (AZA). The mice were administered azacitidine $(2.5 \mathrm{mg} / \mathrm{kg})$ or NS once a day from day 1 for seven consecutive days. The untreated control received NS, drugs and NS were injected intraperitoneally. The weight loss and survival times of the mice were recorded and analyzed. The status of injected SKM-1 cells in peripheral blood was confirmed by Wright staining. All procedures were conducted in accordance with Guidelines for the Care and Use of Laboratory Animals. The protocol was approved by the Committee on the Ethics of Animal Experiments of Guiyang Medical College.

Statistical analysis. Each experiment or assay was performed at least three times, and representative examples are shown. Data are reported as means \pm SEM. Statistically significant differences between the treated groups were calculated using Student's t-test. Differences were considered statistically significant at $\mathrm{P}<0.05$.

\section{Results}

AZA treatment increases $\mathrm{HO}-1$ expression in SKM-1 cells. MDS cell line SKM-1 and AML cell lines U937, HEL and THP-1 were treated with AZA $(0.5 \mu \mathrm{M})$ for $24 \mathrm{~h}$, the HO-1 expression was detected by real-time PCR. AZA treatment obviously increased HO-1 expression in SKM-1 cells, but not in the three AML cell lines (Fig. 1A). Bone marrow MNCs of 48 MDS patients were collected and divided into a verylow-risk group, a low-risk group, a high-risk group and a very high-risk group according to WHO risk classification criteria (Table I). Real-time PCR results showed that the HO-1 expression level of bone marrow MNCs from high-risk and very high-risk MDS patients exceeded that of very low-risk and low-risk patients (Fig. 1B).

Silencing HO-1 enhances the inhibitory effects of AZA on SKM-1 cell growth. As detected by MTT assay, the growth inhibition rates of SKM-1 cells at $24 \mathrm{~h}$ increased (11, 19, 30 and $51 \%$ ) with rising AZA concentration $(0.2,0.5,0.8$ and $1 \mu \mathrm{M})$ (Fig. 1C). Thus, AZA inhibited the growth of SKM-1 cells in a concentration-dependent manner. After being treated with AZA $(0.2,0.5,0.8$ and $1 \mu \mathrm{M})$ for $24 \mathrm{~h}$, the SKM-1-siHO- 1 cells (transduced with HO-1 siRNA) were more prone to inhibition (growth inhibition rates: 26.50, 40, 58 and 78\%). In contrast, the growth inhibition rates of the blank SKM-1 cells (without any treatment) and SKM-1-vector cells (transduced with empty vector) remained almost unchanged (Fig. 1D). When HO-1 expression was upregulated first by Hemin $(15 \mu \mathrm{mol} / \mathrm{l})$, the growth inhibition rates, especially those of the blank SKM-1 

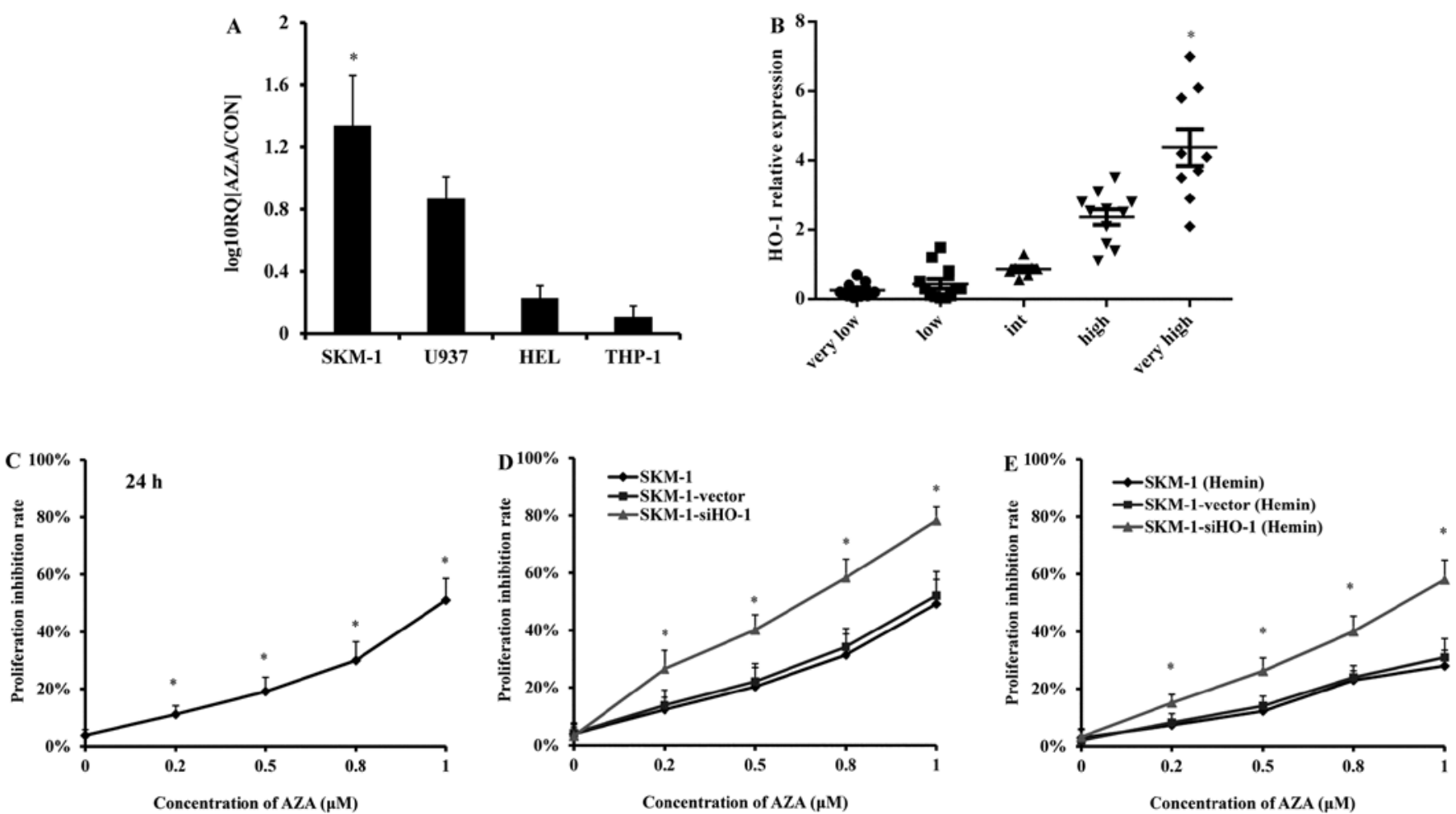

Figure 1. HO-1 relative expression in MDS/AML cell lines and in MDS patients and the inhibitory effects of AZA on SKM-1 cell proliferation. (A) HO-1 expressions in MDS cell line SKM-1 and AML cell line U937, HEL, THP-1 treated with AZA (1 $\mu$ M) by qPCR. (B) HO-1 expressions in MDS patients by qPCR, classified according to the WHO classification. Characteristics of MDS patients are shown in Table I. (C) Effects of AZA $(0.2,0.5,0.8$ and $1 \mu \mathrm{M})$ on cell growth in SKM-1 (untreated) cells. Cells were treated with AZA for $24 \mathrm{~h}$. (D) Effects of AZA (0.2, 0.5, 0.8 and $1 \mu \mathrm{M})$ on cell growth in SKM-1 (control), SKM-1-vector (transfected with empty vector), SKM-1-siHO-1 (transfected with lentivirus-mediated HO-1 siRNA) cells. Cells were treated with AZA for 24 h. (E) Effects of AZA combined with Hemin on cell growth in SKM-1 (control), SKM-1-vector, SKM-1-siHO-1. Cells were treated with Hemin for 24 h, and then treated with AZA for $24 \mathrm{~h}$. Cell viability was measured by MTT. The MTT uptake and the effect were analyzed by Prism V5.0 (GraphPad Software, San Diego, CA, USA). Statistical differences were calculated by one-way ANOVA. *P<0.05.

and SKM-1-vector cells, were apparently decreased after treatment with AZA at the concentrations mentioned above for $24 \mathrm{~h}$ (Fig. 1E). Therefore, the growth inhibitory effect of AZA on SKM-1 cells was attenuated by HO-1 expression and boosted by silencing HO-1 with siRNA.

HO-1 silencing sensitizes SKM-1 cells to AZA-induced apoptosis through the caspase-3-dependent pathway. Given that HO-1 expression resisted AZA-induced inhibition of SKM-1 cell growth, the apoptosis of SKM-1 cells in which HO-1 was silenced by siRNA or upregulated by Hemin was detected by flow cytometry after $24 \mathrm{~h}$ of treatment with $1 \mu \mathrm{M}$ AZA. Compared with the blank control and empty vector group (25.90 and 28.08\%), the apoptotic rate of the HO-1 silencing SKM-1 cells increased remarkably (48.64\%), which, however, plummeted due to HO-1 expression induced by Hemin (Fig. 2A). Hence, HO-1 expression protected SKM-1 cells from AZA-induced apoptosis. To further analyze the mechanism for enhanced apoptosis, the expressions of caspase- 3 and -9 , cleaved caspase- 3 and -9, BCL-2 and Bax were detected by western blot analysis. After treatment with AZA for $24 \mathrm{~h}$, the expression levels of cleaved caspase- 3 and -9 and Bax increased in the HO-1 silencing SKM-1 cells, whereas that of BCL-2 decreased (Fig. 2B). To prove that increase of SKM-1 cell apoptosis was associated with caspase-3-dependent apoptotic pathways, we treated SKM-1 cells with the caspase-3 inhibitor Z-DEVE-FMK as well as AZA for $24 \mathrm{~h}$, and then we evaluated the cell apoptosis by flow cytometry. SKM-1 cells, especially for the HO-1 silencing group, were dramatically less subjected to apoptosis when Z-DEVE-FMK was used (Fig. 2C).

AZA arrests more SKM-1 cells in the G0/G1 phase by silencing $\mathrm{HO}-1$. By regulating the methylation levels of promoters of cell cycle negative regulatory genes such as p15 and p16, AZA facilitates the expressions of p15 and p16 genes and influences the regulation of cell cycle as a result. To explore whether HO-1 affected the cell cycle, flow cytometry was used for the blank control, empty vector and HO-1 silencing SKM-1 cells before and after treatment with AZA or AZA plus Hemin. The results revealed that AZA-induced SKM-1 cell apoptosis was accompanied by G0/G1 arrest, particularly when HO-1 was silenced. On the contrary, HO-1 expression induced by Hemin accelerated the cell cycle progression to G2/M phase (Fig. 3), probably by promoting cell proliferation and differentiation $(15,16)$.

P16 overexpression mediates G0/G1 arrest of SKM-1 cells. Decrease of cell apoptosis and malignant proliferation, which are bound to occur during malignant progression of MDS, are associated with silencing of anti-oncogenes and activation of oncogenes that are dominantly controlled by aberrant meth- 

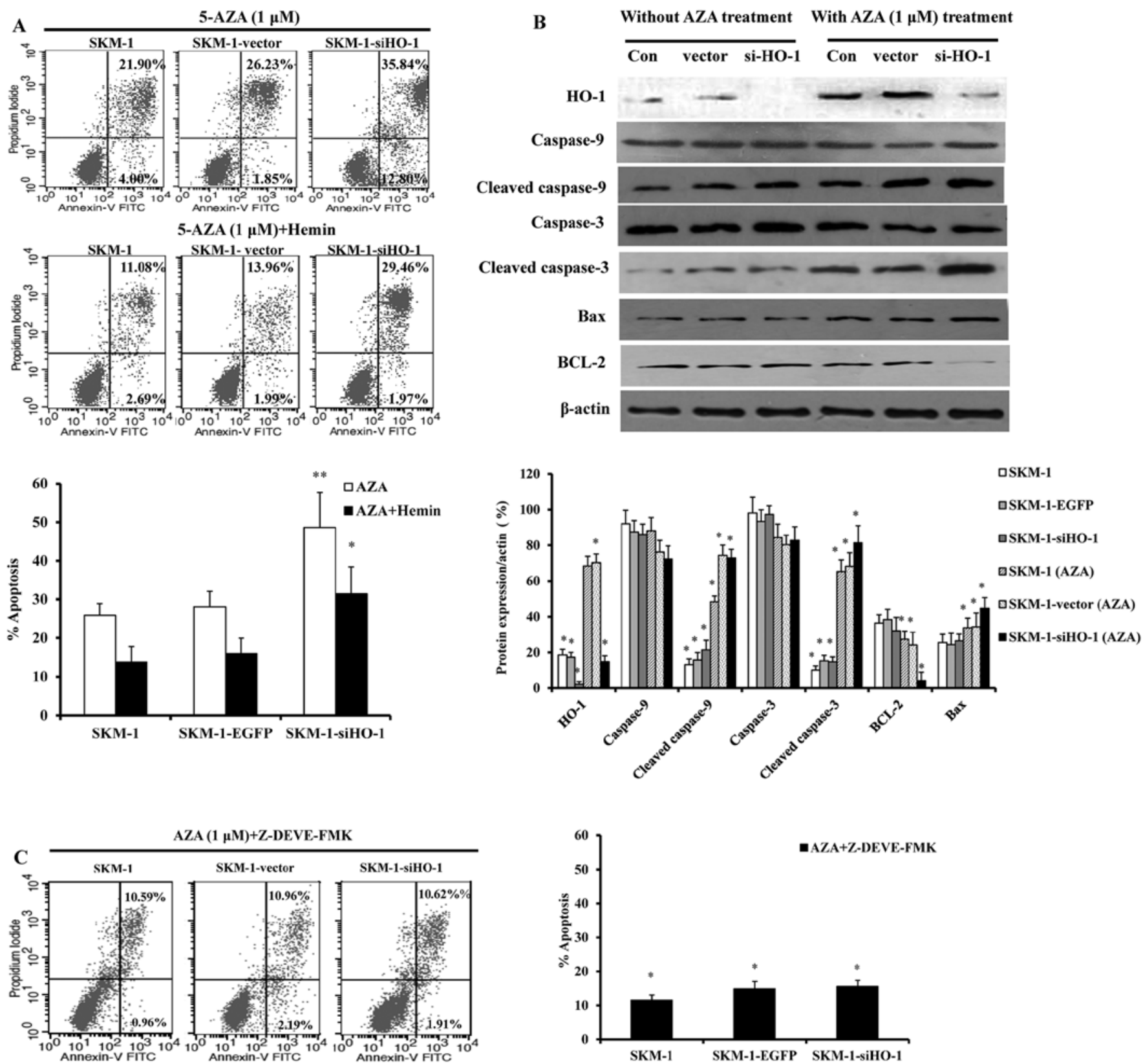

Figure 2. Silencing HO-1 sensitizes SKM-1 cells to apoptosis induced by AZA. SKM-1 cells were grouped into SKM-1 (control), SKM-1-vector, SKM-1-siHO-1. (A) The cells were cultured in the presence of AZA or AZA plus Hemin and apoptosis was analyzed $24 \mathrm{~h}$ thereafter by flow cytometry. Annexin-V-FITC/ PI-positive cells were determined by flow cytometry. Bar graph indicates the percent of Annexin V-positive cells (apoptotic cells). (B) Western blot analysis of apoptosis proteins in SKM-1 cells. Con, SKM-1 (untreated); vector, SKM-1-vector; siHO-1, SKM-1-siHO-1 with or without AZA (1 $\mu$ M) treatment. Protein expressions of HO-1, caspase- $9,-3$, cleaved caspase- $9,-3, \mathrm{Bcl}-2$ and BAX, are depicted. Western blot bands were quantified with Quantity One software. Each sample was normalized by related $\beta$-actin expression. (C) SKM-1 (untreated), SKM-1-vector, SKM-1-siHO-1 cells were added to caspase-3 inhibitor combined with AZA, apoptosis was analyzed $24 \mathrm{~h}$ thereafter by flow cytometry. Bar graph indicates the percent of Annexin V-positive cells (apoptotic cells). All measurements were conducted in triplicate. ${ }^{*} \mathrm{P}<0.05,{ }^{* *} \mathrm{P}<0.01$.

ylation $(17,18)$. To clarify whether AZA-induced significant HO-1-silencing SKM-1 cell proliferation inhibition, apoptosis increase and G0/G1 arrest were associated with regulation of methylation-related genes, the expressions of such genes (TP53, RUNX1, NRAS, KRAS, TET2, ASXL1, CBL, EZH2, IDH1, IDH2, DNMT1, DNMT3A, SF3B1, BCL-2, p15, p16 and HDAC) in AZA- or/and Hemin-treated SKM-1 cells were detected by real-time PCR. We found AZA-treated SKM-1 cells showed decreased DNMT1, BCL-2 expression and increased p16, p15 expression, of these genes, DNMT1, BCL-2 and 16 showed significant changes. However, HO-1 expression induced by Hemin evidently decreased such effects (Fig. 4A). Moreover, after lentivirus-mediated HO-1 knockdown and treatment with $1 \mu \mathrm{M}$ AZA for $24 \mathrm{~h}$, compared with treatment with AZA alone, the expression of DNMT1 and BCL-2 was further reduced and p16 further increased (Fig. 4B). We also found that HO-1 silenced SKM-1 cells tended to arrest in the G0/G1 phase after treatment with AZA, for which increased expression of p16 may be responsible. Next, we detected the relative expression of cell cycle-related genes (CDK4, CDK6, 
A

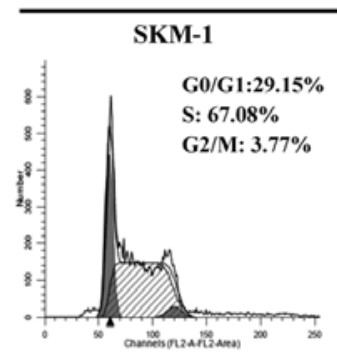

5-AZA $(1 \mu \mathrm{M})$

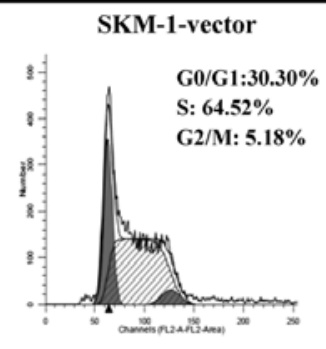

5-AZA $(1 \mu \mathrm{M})+$ Hemin

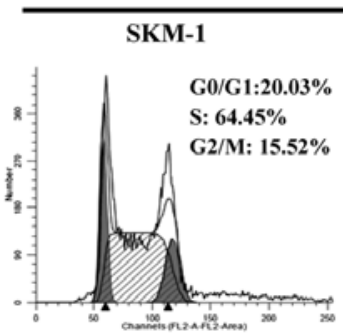

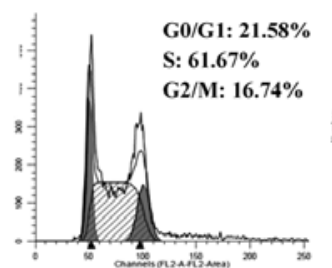

SKM-1-vector
SKM-1-siHO-1

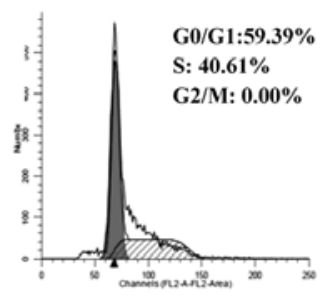

SKM-1-siHO-1

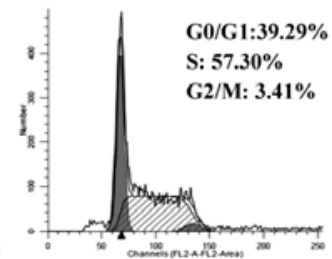

B
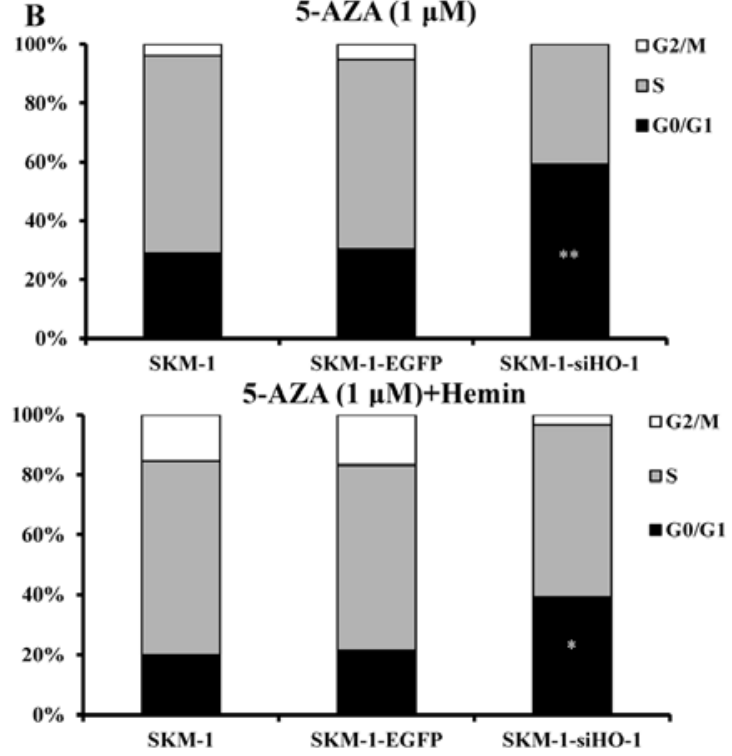

Figure 3. Effects of low concentration AZA on cell cycle progression of SKM-1 after silencing HO-1. SKM-1 cells were grouped into SKM-1 (untreated), SKM-1-vector and SKM-1-siHO-1. (A) SKM-1 cells were cultured with AZA or AZA plus Hemin and cell cycle was analyzed by flow cytometry after 24 h.

(B) Graphs show the average percentage of cells in each phase of the cell cycle. Three separate experiments were performed.
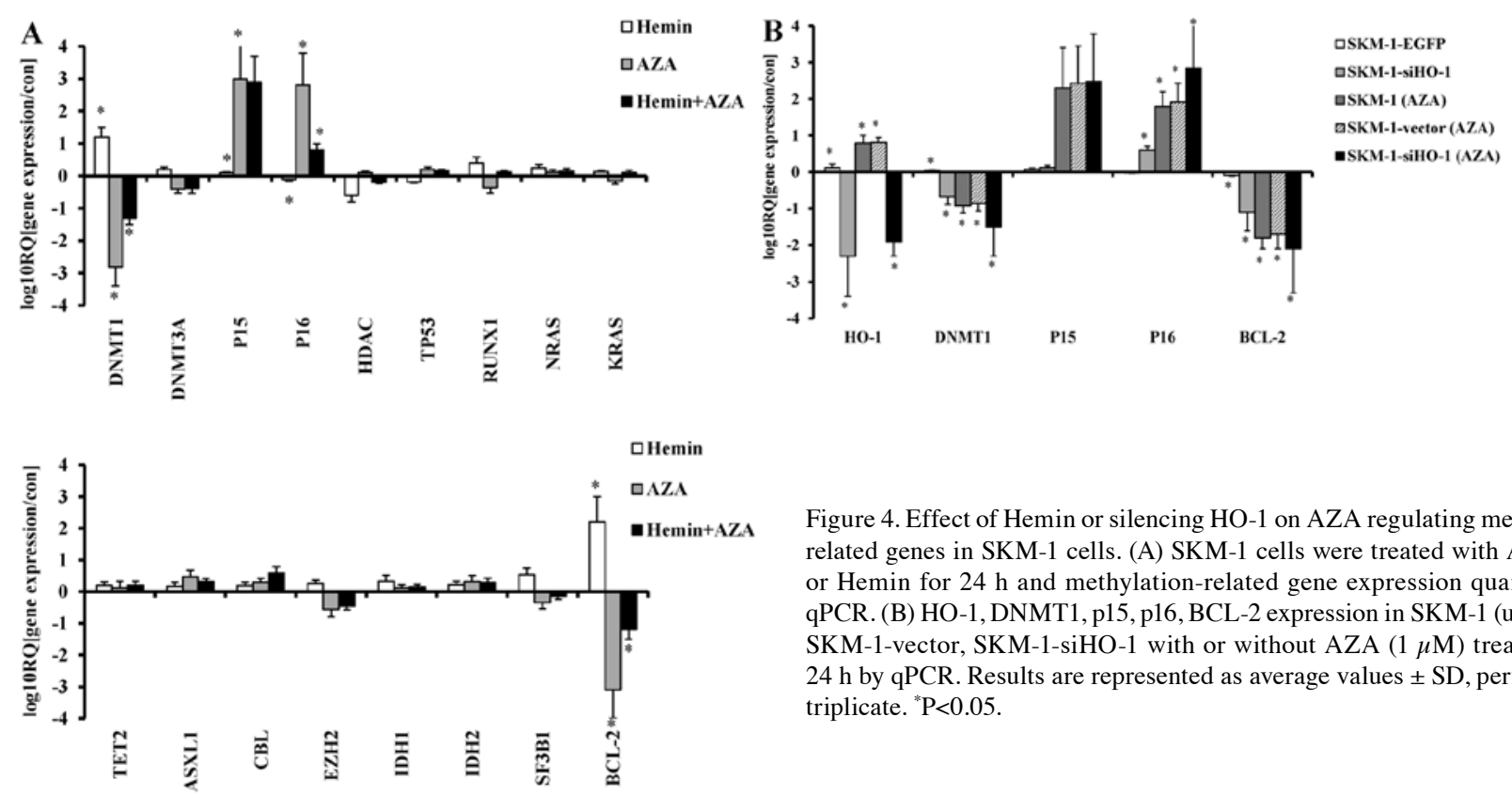

Figure 4. Effect of Hemin or silencing HO-1 on AZA regulating methylationrelated genes in SKM-1 cells. (A) SKM-1 cells were treated with AZA and/ or Hemin for $24 \mathrm{~h}$ and methylation-related gene expression quantified by qPCR. (B) HO-1, DNMT1, p15, p16, BCL-2 expression in SKM-1 (untreated), SKM-1-vector, SKM-1-siHO-1 with or without AZA $(1 \mu \mathrm{M})$ treatment for $24 \mathrm{~h}$ by qPCR. Results are represented as average values $\pm \mathrm{SD}$, performed in triplicate. ${ }^{*} \mathrm{P}<0.05$.

Cyclin D1, Cyclin E, p21, p27, P57,pRB and E2F1) by real-time PCR, silencing HO-1 further reduced CDK4, CDK6, pRB, E2F1 expressions induced by AZA, whereas p27 expression further increased (Fig. 5A). Western blot analysis showed the protein expressions of p16, CDK4, CDK6, pRB, E2F1 and p27 changed in accordance with the above real-time PCR results (Fig. 5B).

HO-1 silencing enhances the demethylating effect of AZA on pl6 gene promoter and subsequently promoted pl6 expression through inhibiting DNMT1. Given that HO-1 silencing increases p16 expression induced by AZA in SKM-1 cells,

we analyzed whether HO-1 promoted p16 expression by influencing its demethylation level through MSP. Compared with SKM-1 (without treatment) and SKM-1-vector cells (transfected with empty vector), the demethylation level of p16 in SKM-1-siHO-1 cells (transfected with HO-1 siRNA) did not change obviously, which was then augmented significantly after treatment with AZA for $24 \mathrm{~h}$ (Fig. 6A). However, when HO-1 was upregulated by Hemin, the demethylating effects of AZA, as suggested by MSP, were diminished (Fig. 6B). We have found that silencing $\mathrm{HO}-1$ facilitated AZA-induced p16 expression, then we blocked DNMT1 gene by DNMT1 siRNA and upregulated HO-1 expression by Hemin at the same time, 


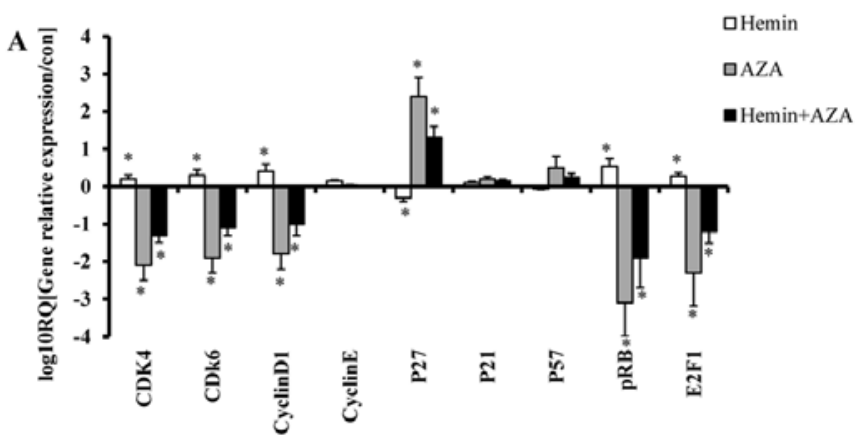

B Without AZA treatment With AZA $(1 \mu M)$ treatment \begin{tabular}{lllll}
\hline Con Vector si-HO-1 & Con Vector & si-HO-1
\end{tabular}
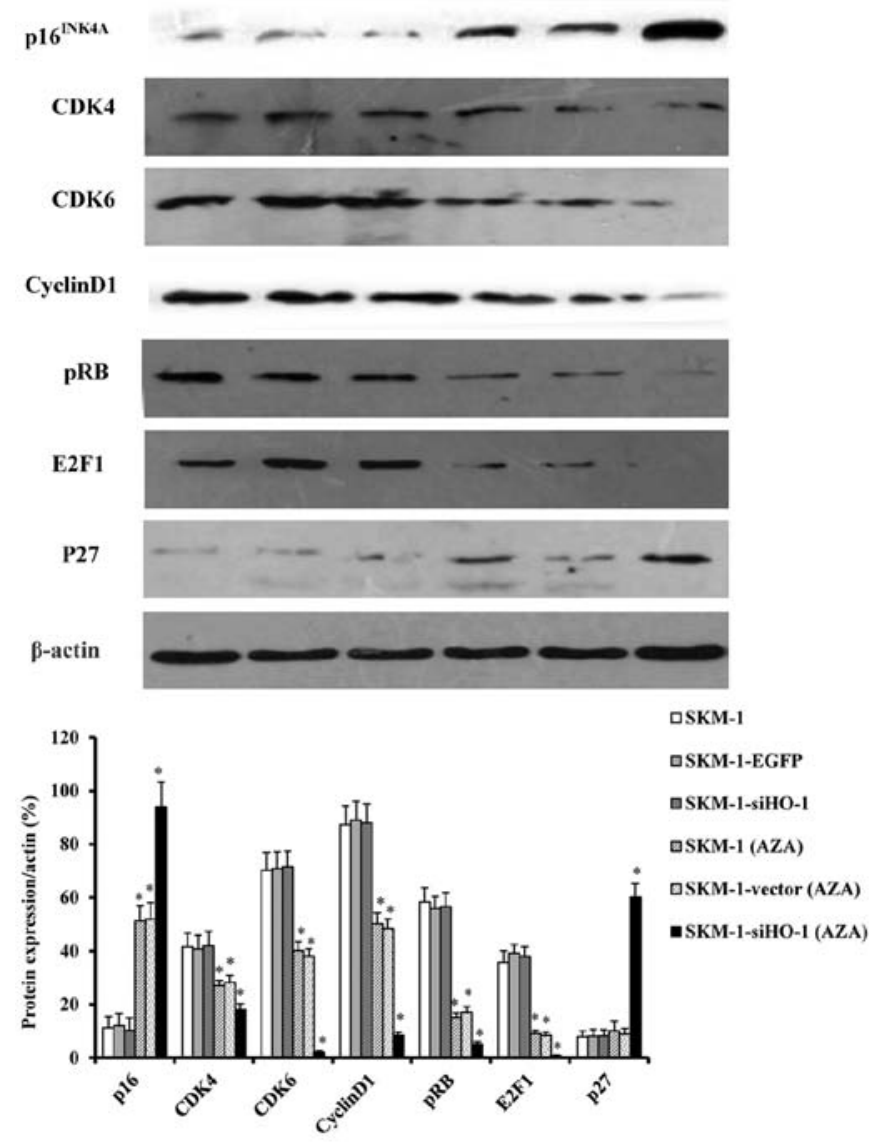

Figure 5. Effects of Hemin or silencing HO-1 on AZA regulating cell cycle related genes in SKM-1 cells. (A) SKM-1 cells were treated with AZA and/or Hemin for $24 \mathrm{~h}$ and cell cycle related gene expression quantified by qPCR. (B) Con, SKM-1 (untreated); vector, SKM-1-vector; si-HO-1, SKM-1-siHO-1 with or without AZA $(1 \mu \mathrm{M})$ treatment. The cell cycle regulatory proteins were assessed by western blot analysis in SKM-1 cells. $\beta$-actin served as a loading control. The blot is representative for of least 3 separate experiments. ${ }^{*} \mathrm{P}<0.05$.

western blot analysis confirmed HO-1 induction promoted DNMT1 expression, however, when blocking DNMT1 by siRNA at the same time, DNMT1 expression significantly decreased, and p16 showed a relatively high protein expression level (Fig. 6C).

Silencing HO-1 efficiently enhances the effects of AZA on inhibiting SKM-1 cells in intravenous MDS mouse model.
In vivo, we established the intravenous MDS mouse model through injecting SKM-1 (without any treatment), SKM-1vector (transfected with empty vector), SKM-1-siHO-1 (transfected with lentivirus-mediated HO-1 siRNA) cells. Treatment of the MDS mice with $2.5 \mathrm{mg} / \mathrm{kg}$ azacitidine resulted in significant suppression of SKM-1 cell growth, especially for the HO-1 silenced SKM-1 cells. On day 14, the AZA-treated groups: SKM-1 (AZA), SKM-1-vector (AZA), SKM-1-siHO-1 (AZA) weighed, respectively 92, 91.4 and $93 \%$ of the mean body weight of the normal NOD/SCID mice, the NS-treated groups: SKM-1, SKM-1-vector and SKM-1siHO-1 weighed, respectively $56,57.4$ and $60 \%$ of the mean body weight of the normal NOD/SCID mice (Fig. 7A). We observed that the overall survival of AZA-treated MDS mice was prolonged, and in NS-treated MDS mice it shortened (Fig. 7B). Wright-staining analysis revealed a relatively lower number of primitive SKM-1 cells in AZA-treated MDS mice compared with the NS-treated in peripheral blood, and we found that the AZA-treated MDS mice injected the SKM-1siHO-1 cells showed the lowest number of the primitive SKM-1 cells (Fig. 7C). In addition, flow cytometry showed decreased human CD45 ${ }^{+}$SKM-1 cells in the peripheral blood of AZA-treated MDS mice, the AZA-treated SKM-1-siHO-1 mice, however, showed the most significant decrease of human CD45 ${ }^{+}$cells (Fig. 7D).

\section{Discussion}

Epigenetic changes, in addition to genetic mutation, predominantly control the onset of MDS/AML. As a common epigenetic inhibitor, AZA, when inserted into newly synthesized DNA, can demethylate DNA by binding DNA methyltransferase irreversibly. It inhibits the synthesis of proteins after being inserted into RNA (5). Besides, AZA is able to regulate cell differentiation and proliferation (19). AZA was approved by FDA to treat all MDS subtypes in 2004, and by using them Kumode et al successfully treated MDS-derived AML (20). Despite the significantly elevated overall survival rates of medium-risk II and high-risk MDS patients, AZA usually gives unsatisfactory complete remission rates. Some patients are insensitive to AZA, and the mechanism remains unknown. Moreover, the therapeutic effects of AZA may depend on gender, and females tend to have higher plasma AZA concentrations than those of males and thus enjoy slower disease progression (21). If found and regulated in time, the latent adverse factors no longer hinder AZA to exert effects, which are beneficial to the treatment of high-risk and very high-risk MDS. Recently, HO-1 has been implicated in many solid tumor-related studies, and high HO-1 expression may suggest progression, poor prognosis and chemotherapy resistance of leukemia (11). For patients receiving chemotherapy, high HO-1 expression can both protect normal bone marrow cells and result in resistance of tumor cells (12). By regulating transcription factors Nrf2, NF- $\mathrm{kB}$ and AP-1 and by decreasing ROS accumulation in AML cells, HO-1 shields the cells from TNF-induced apoptosis (22). Rushworth et al and Rushworth and MacEwan reported that Fas-associated death domainlike interleukin $1 \beta$-converting enzyme-like inhibitory protein (FLIP) promoted AML cells to resist apoptosis by regulating HO-1 expression $(10,11)$. There are no research reports on the 
A
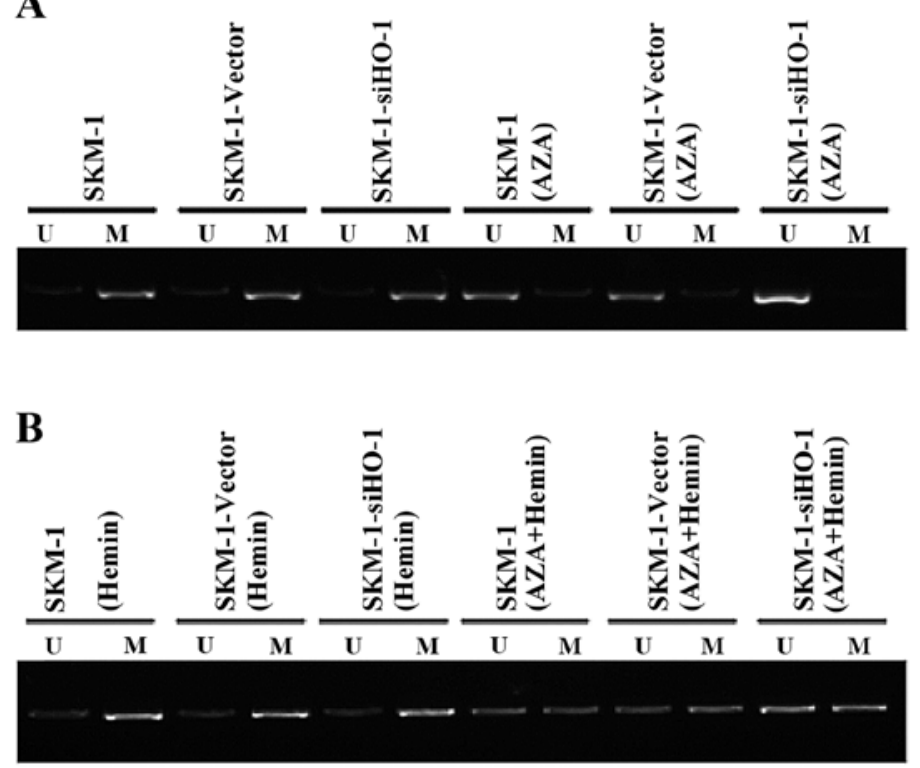

C
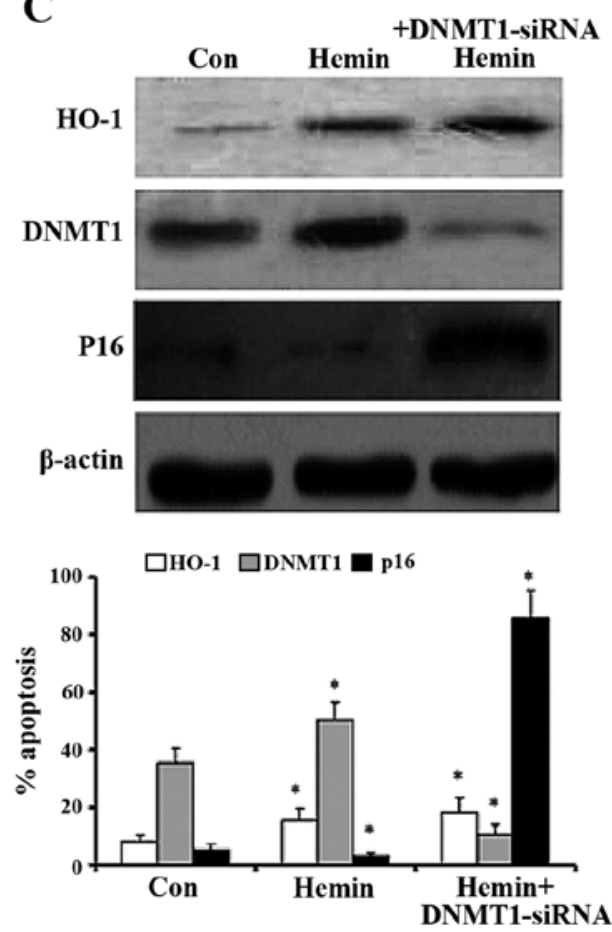

Figure 6. Effects of silencing HO-1 or Hemin on AZA demethylating p16 promoter and the influence of HO-1 on p16 expression when blocking DNMT1. (A) MSP to identify changes in the methylation status at p16 promoter in SKM-1 cells (control), SKM-1-vector (transfected with empty vector), SKM-1-siHO-1 (transfected with lentivirus-mediated HO-1 siRNA) cells treated with or without AZA (1 $\mu$ M) for 24 h. (B) MSP to identify changes in the methylation status at p16 promoter in SKM-1 cells (control), SKM-1-vector (transfected with empty vector), SKM-1-siHO-1 (transfected with lentivirus-mediated HO-1 siRNA) cells treated with Hemin or AZA plus Hemin. Primers were designed to study DNA methylation patterns in CpG islands of the p16 promoter start site. The presence of a PCR product in lanes labeled U indicates an unmethylated status for the p16 ${ }^{\text {INK4a }}$ promoter region, whereas the presence of a PCR product in lanes labeled M indicates a methylated promoter status. (C) Western blot analysis of the effects of HO-1 or HO-1 combined with blocking DNMT1 by DNMT1 siRNA on DNMT1 and p16 expression. Western blot bands were quantified with Quantity One software. Each sample was normalized by related $\beta$-actin expression. Repeated in triplicate. ${ }^{*} \mathrm{P}<0.05$.

role of HO-1 in AZA treatment. HO-1 counteracts the therapeutic effects of AZA on MDS via a yet unknown mechanism. In this study, we silenced HO-1 by lentivirus-mediated siRNA or upregulated HO-1 by Hemin to evaluate the influences of AZA on the proliferation, apoptosis, and cell cycle of SKM-1 cells in vitro and in vivo, and to explore the possible mechanism.

MDS/AML cell lines SKM-1, HEL, U937 and THP-1 were treated with AZA $(0.5 \mu \mathrm{M})$ for $24 \mathrm{~h}$, and bone marrow MNCs from 48 MDS patients were collected. HO-1 expressions were then detected by real-time PCR. Compared with AML cell lines HEL, U937 and THP-1, SKM-1 cells expressed higher level of HO-1, which was further increased by AZA treatment. In contrast, AZA treatment barely varied the expression levels of HO-1 in AML cells. In addition, the HO-1 expression levels of bone marrow MNCs from high-risk and very high-risk MDS patients exceeded those of low-risk and very low-risk patients, implying that HO-1 may be associated with MDS malignant progression.

Subsequently, we silenced HO-1 by lentivirus-mediated siRNA or upregulated HO-1 by Hemin to evaluate the roles of HO-1 in the AZA-induced growth inhibition, apoptosis and cell cycle arrest of SKM-1 cells. MTT assay showed that AZA suppressed the proliferation of SKM-1 cells concentrationdependently, and that the inhibitory effects were drastically boosted by silencing HO-1 but were diminished by Hemin.
However, the HO-1 silencing SKM-1 cells were less prone to Hemin treatment, probably because Hemin could not induce HO-1 expression as effectively as it usually did when HO-1 was silenced by siRNA. After HO-1 was silenced, flow cytometry exhibited that AZA induced significant apoptosis of SKM-1 cells. When HO-1 was upregulated by Hemin, however, we found the apoptosis effects induced by AZA were weaken, the blank control and empty vector SKM-1 cells underwent significantly less apoptosis than the HO-1 silencing SKM-1 cells did, similar outcomes to those of MTT assay were obtained. Furthermore, flow cytometry showed that AZA arrested SKM-1 cells in the G0/G1 phase, particularly when HO-1 was silenced. Upregulating HO-1 by Hemin accelerated cell cycle progression to the G2/M phase, which may be ascribed to the facilitated cell proliferation and differentiation by $\mathrm{HO}-1$ (15). By using real-time PCR, the expressions of methylationrelated genes in AZA- or/and Hemin-treated SKM-1 cells [RNA splicing gene (SF3B1), DNA methylation genes (TET2, DNMT1, DNMT3A, IDH1 and IDH2), chromatin-modifying genes (ASXL1 and EZH2), transcriptional regulatory gene (RUNX1), DNA repair gene (TP53), signal transduction genes (CBL, NRAS and KRAS), histone acetylation gene (HDAC), negative cell cycle regulatory genes (p15 and p16), and apoptosis regulatory gene (BCL-2)] were detected (23). Of all the genes, only DNMT1, p16 and BCL-2 were subjected to expression changes. With HO-1 upregulation, the expression 


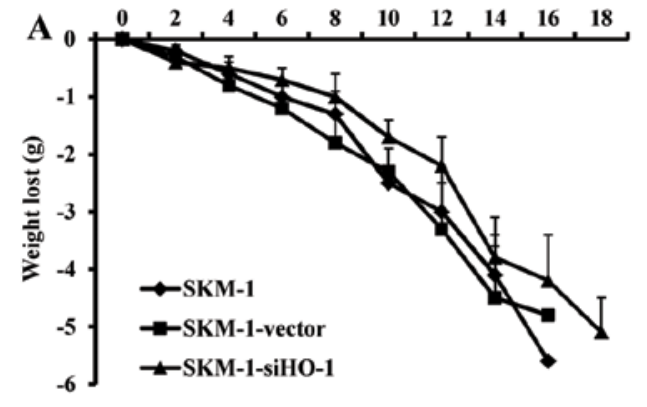

Days after transplantation

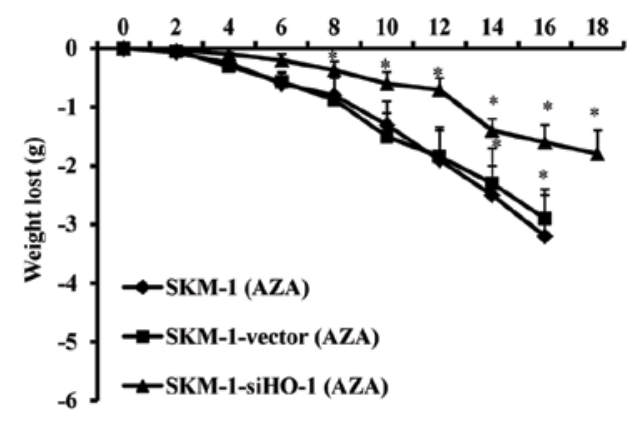

Days after transplantation
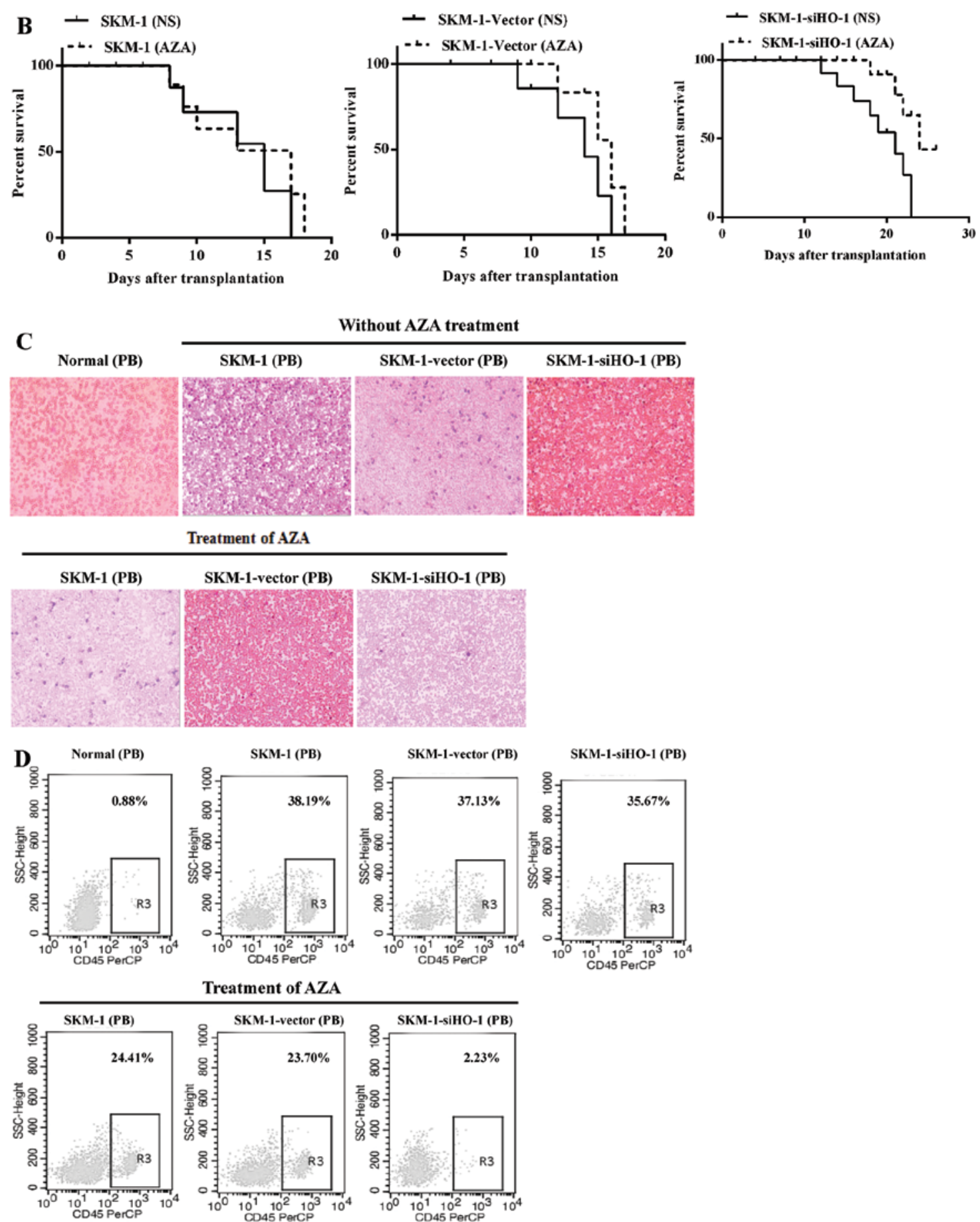

Treatment of AZA
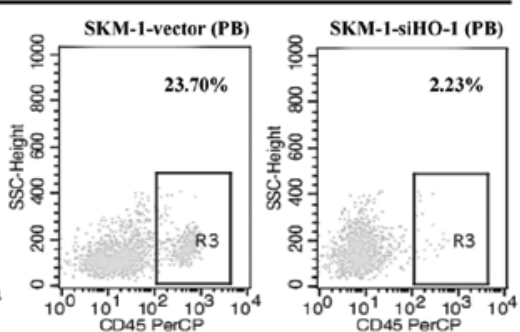

Figure 7. Silencing HO-1 significantly sensitized AZA to inhibit SKM-1 cells in vivo. (A) Weight lost for MDS mice after transplantation. (B) Kaplan-Meier survival curves for recipients of SKM-1 (control), SKM-1-vector (transfected with empty vector), SKM-1-siHO-1 (transfected with lentivirus-mediated HO-1 siRNA) cells. The recipients of the three groups were futher grouped into two according to AZA treatment or not. (C) Peripheral blood smears showed accumulation of SKM-1 cells, indicating SKM-1 cell counts in these MDS mice. Original magnification, x400. (D) FACS analysis showed appearance of human $\mathrm{CD} 45^{+}$cells in peripheral blood of MDS or normal mice. ${ }^{*} \mathrm{P}<0.05$. 
of DNMT1 and BCL-2 increased, while that of p16 decreased. Based on the above experiments, the expressions of HO-1, DNMT1, p16 and BCL-2 genes were detected again in HO-1 silencing SKM-1 cells with or without AZA, the expression of p16 further increased, and DNMT1, BCL-2 further decreased. BCL-2 is a target for sensitizing AZA, specific inhibition of BCL-2 contributes greatly to the growth inhibitory effects of AZA on AML cell lines TF-1 and ML-2 (24). BCL2L10 is a predictive factor which can predict whether or not a patient will become resistant to AZA (25), so we considered the increased apoptosis of SKM-1 cells was related to BCL-2 downregulation. p16 gene is methylated upon MDS or AML (26). Its high expression in low-risk MDS patients may be associated with ineffective hematopoiesis that results from p16-mediated cell senescence, while its low expressions in high-risk MDS patients may be related with MDS malignant progression (27). Moreover, p16 arrests the T-ALL cell line CCRF-CEM in the G0/G1 phase via the pRB-E2F1 pathway (28). Therefore, we largely attributed HO-1 silencing-enhanced sensitivity of AZA to increase in p16 expression.

In gastric cancer, restoration of p16 suppressed CDK4, Cyclin D1 and delayed cell cycle transition (29). Real-time PCR showed that of all the cell cycle-related genes (CDK4, CDK6, Cyclin D1, Cyclin E, p21, p27, p57, pRB and E2F1), the levels of AZA-induced CDK4, CDK6, pRB and E2F1 expression plummeted after HO-1 was silenced, whereas, that of p27 expression increased and those of Cyclin E and p21 remained almost unchanged. Moreover, silencing HO-1 and treating SKM-1 cells with AZA for $24 \mathrm{~h}$ simultaneously, as indicated by western blot analysis, augmented p16, p27 expression and reduced the expression of CDK4, CDK6, pRB and E2F1, suggesting that G0/ G1 arrest was related with p16 expression. In our study, however, we did not use specific p16 inhibitor or p16 siRNA to intervene with its expression, so, it remains further to be confirmed.

$\mathrm{P} 27 / \mathrm{CDKN} 1 \mathrm{~B}$ is known to mediate the cell cycle exit with differentiation (30), so p27 may be involved in G0/G1 exit due to AZA-induced differentiation. By using MSP, we analyzed whether HO-1 promoted the p16 expression by influencing its demethylation level. After HO-1 was silenced, AZA demethylated p16 much more effectively, as evidenced by the apparently increased demethylation products. It has been reported that PM exposure increased ROS production, DNMT1 expression and methylation of the p16 promoter, and finally increased the risk of lung cancer (31). Our study revealed that AZA suppressed DNMT1 more evidently and p16 expression further increased after silencing HO-1. In order to determine how HO-1 affected p16 expression, we blocked the DNMT1 by DNMT1 siRNA and found that hemin-induced HO-1 expression can promote DNMT1 expression and inhibit p16 expression slightly, and blocking DNMT1 at the same time, DNMT1 decreased and p16 increased significantly, suggesting that HO-1 may inhibit p16 expression through promoting DNMT1 expression. However, HO-1 alone showed sight effects on p16 expression, silencing HO-1 just sensitized AZA to facilitate p16 expression.

Furthermore, the expression levels of caspase-3, cleaved caspase-3, caspase-9, cleaved caspase-9, BCL-2 and Bax proteins in AZA-treated SKM-1-siHO-1 cells were detected. The expressions of caspase- 3 and -9 fluctuated mildly, and those of cleaved caspase- 3 and -9 increased, the ratio of BCL-2/BAX decreased. To further determine the apoptosis- related mechanism, caspase-3 inhibitor, Z-DEVE-FMK, was added, after which the apoptotic rate was, as detected by flow cytometry, significantly reduced. When caspase-3 was inhibited, its activated form, cleaved caspase-3, also decreased. In myeloid (P39, HL60) and T cells (Jurkat), AZA induced apoptosis via multiple and separately regulated pathways, such as cleavage of Bcl-2 family proteins, activation of caspase-2 and -3-like, and induction of hypomethylation (32). Thus, we concluded that the AZA-induced apoptosis may be correlated with caspase-3-dependent pathway.

In vivo, silencing HO-1 efficiently enhances the effects of AZA on inhibiting SKM-1 cells with no significant weight decrease, prolonging survival time and reduction in SKM-1 cells in peripheral blood. These results further confirmed that silencing HO-1 played a crucial role in enhancing the AZA-induced apoptosis.

In conclusion, our study revealed that silencing HO-1 sensitized SKM-1 cells to AZA in vitro and in vivo. After being treated with AZA, SKM-1 cells expressed more HO-1, and the bone marrow MNCs from high-risk and very high-risk MDS patients had higher HO-1 expression than those from low-risk and very low-risk patients. With HO-1 silenced, AZA began to inhibit the proliferation of SKM-1 cells more potently, accompanied by raised apoptotic rate and dominant arrest in the G0/G1 phase. The changes were related with increases in the expressions of p16, cleaved caspase- 3 and -9 as well as decrease in BCL-2/Bax ratio. Hence, $\mathrm{HO}-1$ may be one of the targets that sensitize AZA to fight against MDS malignant progression, which paves the way for treating high-risk and very high-risk MDS in clinical practice.

\section{Acknowledgements}

This study was supported, in part, by the National Natural Science Foundation of China (nos. 81070444, 81270636, 81360501 and 81470006), International Cooperation Project of Guizhou Province (no. 2011-7010), Social Project of Guizhou Province (no. 2011-3012), Provincial Government Special Fund of Guizhou Province (no. 2010-84), and Project of Science and Technology Bureau of Guiyang City (no. [2012103-36]).

\section{References}

1. Sekeres MA and Cutler C: How we treat higher-risk myelodysplastic syndromes. Blood 123: 829-836, 2014.

2. Jiang Y, Dunbar A, Gondek LP, et al: Aberrant DNA methylation is a dominant mechanism in MDS progression to AML. Blood 113: $1315-1325,2009$

3. Stintzing S, Kemmerling R, Kiesslich T, Alinger B, Ocker M and Neureiter D: Myelodysplastic syndrome and histone deacetylase inhibitors: 'to be or not to be acetylated'? J Biomed Biotechnol 2011: 214143, 2011.

4. Fenaux P, Mufti GJ, Hellstrom-Lindberg E, et al: Efficacy of azacitidine compared with that of conventional care regimens in the treatment of higher-risk myelodysplastic syndromes: a randomised, open-label, phase III study. Lancet Oncol 10: 223-232, 2009.

5. Kimura S, Kuramoto K, Homan J, et al: Antiproliferative and antitumor effects of azacitidine against the human myelodysplastic syndrome cell line SKM-1. Anticancer Res 32: 795-798, 2012.

6. Figueroa ME, Skrabanek L, Li Y, et al: MDS and secondary AML display unique patterns and abundance of aberrant DNA methylation. Blood 114: 3448-3458, 2009.

7. Ades L and Santini V: Hypomethylating agents and chemotherapy in MDS. Best Pract Res Cl Ha 26: 411-419, 2013. 
8. Khan C, Pathe N, Fazal S, Lister J and Rossetti JM: Azacitidine in the management of patients with myelodysplastic syndromes. Ther Adv Hematol 3: 355-373, 2012.

9. Jozkowicz A, Was H and Dulak J: Heme oxygenase-1 in tumors: is it a false friend? Antioxid Redox Sign 9: 2099-2117, 2007.

10. Rushworth SA, Zaitseva L, Langa S, Bowles KM and MacEwan DJ: FLIP regulation of HO-1 and TNF signalling in human acute myeloid leukemia provides a unique secondary anti-apoptotic mechanism. Oncotarget 1: 359-366, 2010.

11. Rushworth SA and MacEwan DJ: HO-1 underlies resistance of AML cells to TNF-induced apoptosis. Blood 111: 3793-3801, 2008.

12. Ma D, Fang Q, Li Y, et al: Crucial role of heme oxygenase-1 in the sensitivity of acute myeloid leukemia cell line Kasumi-1 to ursolic acid. Anti-cancer Drug 25: 406-414, 2014.

13. Su ZY, Shu L, Khor TO, Lee JH, Fuentes F and Kong AN: A perspective on dietary phytochemicals and cancer chemoprevention: oxidative stress, nrf2, and epigenomics. Topics Curr Chem 329: 133-162, 2013

14. Kang KA, Piao MJ, Kim KC, et al: Epigenetic modification of Nrf2 in 5-fluorouracil-resistant colon cancer cells: involvement of TET-dependent DNA demethylation. Cell Death Dis 5: e1183, 2014.

15. Zhang LF, Qi J, Zuo G, et al: Osteoblast-secreted factors promote proliferation and osteogenic differentiation of bone marrow stromal cells via VEGF/heme-oxygenase-1 pathway. PLoS One 9: e99946, 2014

16. Wegiel B, Hedblom A, Li M, et al: Heme oxygenase-1 derived carbon monoxide permits maturation of myeloid cells. Cell Death Dis 5: e1139, 2014.

17. Parker JE, Mufti GJ, Rasool F, Mijovic A, Devereux S and Pagliuca A: The role of apoptosis, proliferation, and the Bcl-2-related proteins in the myelodysplastic syndromes and acute myeloid leukemia secondary to MDS. Blood 96: 3932-3938, 2000.

18. Issa JP: The myelodysplastic syndrome as a prototypical epigenetic disease. Blood 121: 3811-3817, 2013.

19. Curik N, Burda P, Vargova K, et al: 5-azacitidine in aggressive myelodysplastic syndromes regulates chromatin structure at PU.1 gene and cell differentiation capacity. Leukemia 26: 1804-1811, 2012.

20. Kumode T, Fukui A, Eguchi G, Yamaguchi T and Maeda Y: A case of secondary leukemia subsequent to myelodysplastic syndromes successfully treated with azacitidine. Case Rep Med 2014: 793928, 2014
21. Jasielec J, Saloura V and Godley LA: The mechanistic role of DNA methylation in myeloid leukemogenesis. Leukemia 28: 1765-1773, 2014

22. Heasman SA, Zaitseva L, Bowles KM, Rushworth SA and Macewan DJ: Protection of acute myeloid leukaemia cells from apoptosis induced by front-line chemotherapeutics is mediated by haem oxygenase-1. Oncotarget 2: 658-668, 2011.

23. Cazzola M, Della Porta mg and Malcovati L: The genetic basis of myelodysplasia and its clinical relevance. Blood 122: 4021-4034, 2013.

24. Bogenberger JM, Kornblau SM, Pierceall WE, et al: BCL-2 family proteins as 5-Azacytidine-sensitizing targets and determinants of response in myeloid malignancies. Leukemia 28: $1657-1665,2014$

25. Cluzeau T, Robert G, Mounier N, et al: BCL2L10 is a predictive factor for resistance to azacitidine in MDS and AML patients. Oncotarget 3: 490-501, 2012.

26. Karlic H, Herrmann H, Varga F, et al: The role of epigenetics in the regulation of apoptosis in myelodysplastic syndromes and acute myeloid leukemia. Crit Rev Oncol Hemat 90: 1-16, 2014.

27. Wang YY, Cen JN, He J, et al: Accelerated cellular senescence in myelodysplastic syndrome. Exp Hematol 37: 1310-1317, 2009.

28. Ausserlechner MJ, Obexer P, Geley S and Kofler R: G1 arrest by p16INK4A uncouples growth from cell cycle progression in leukemia cells with deregulated cyclin $\mathrm{E}$ and $\mathrm{c}-\mathrm{Myc}$ expression. Leukemia 19: 1051-1057, 2005.

29. Kim JK, Noh JH, Eun JW, et al: Targeted inactivation of HDAC2 restores p16INK4a activity and exerts antitumor effects on human gastric cancer. Mol Cancer Res 11: 62-73, 2013.

30. Ng KP, Ebrahem Q, Negrotto S, et al: p53 independent epigenetic-differentiation treatment in xenotransplant models of acute myeloid leukemia. Leukemia 25: 1739-1750, 2011.

31. Soberanes S, Gonzalez A, Urich D, et al: Particulate matter Air Pollution induces hypermethylation of the p16 promoter Via a mitochondrial ROS-JNK-DNMT1 pathway. Sci Rep-UK 2: 275, 2012.

32. Khan R, Schmidt-Mende J, Karimi M, et al: Hypomethylation and apoptosis in 5-azacytidine-treated myeloid cells. Exp Hematol 36: 149-157, 2008 